\title{
A COMPARATIVE STUDY ON 100\% TCA VERSUS 88\% PHENOL FOR THE TREATMENT OF VITILIGO
}

\author{
by Neerja Puri, Asha Puri
}

\author{
comment: \\ Cheng-Che E. Lan, MD, PhD
}

Our Dermatol Online. 2012;3(3): 187

Date of submission: 01.06.2012 / acceptance: 07.06.2012

Conflicts of interest: None

The study performed by Puri and Puri appearing in this issue suggested that TCA and phenol, the two medium depth chemical peeling agents, may be used to induce repigmentation of stable vitiligo lesions. Although different modalities have been introduced for vitiligo treatment, surgical intervention is still considered as an important treatment option for stable vitiligo lesions that do not respond to conventional therapies. Different surgical techniques, some requiring cellular cultures, have been established for treating stable vitiligo. More recently, dermabrasion has also been suggested to be an alternative intervention, especially if followed by phototherapy [1]. While it is of academic importance to clarify if the mechanisms involved in repigmentation differ between these different surgical approaches, it is perhaps even more crucial to identify "stable" vitiligo lesions that will most likely respond to surgical interventions.

\section{REFERENCES}

1. Bayoumi W, Fontas E, Sillard L, Le Duff F, Ortonne JP, Bahadoran P, et al: Effect of a preceding laser dermabrasion on the outcome of combined therapy with narrowband ultraviolet $\mathrm{B}$ and potent topical steroids for treating nonsegmental vitiligo in resistant localizations. Br J Dermatol. 2012; 166: 208-211.

Correspondence:

Cheng-Che E. Lan, MD, PhD

Department of Dermatology, Kaohsiung Medical University Hospital, Kaohsiung, Taiwan.

100 Shih-Chuan 1st Rd. Kaohsiung, Taiwan.

E-mail:laneric@gmail.com 